\title{
The prevalence of dysfunctional breathing in adults in the community with and without asthma
}

\author{
Mike Thomas ${ }^{\mathrm{a}, *}$, Robert K. McKinley ${ }^{\mathrm{b}}$, Elaine Freemanc, \\ Chris Foy ${ }^{d}$, David Price
}

\author{
a GPIAG Clinical Research Fellow, Department of General Practice and Primary Care, University of \\ Aberdeen, UK \\ ${ }^{\mathrm{b}}$ Department of General Practice and Primary Health Care, University of Leicester, UK \\ c Gloucestershire Research and Development Support Unit, Gloucestershire Health Authority, UK \\ ${ }^{\mathrm{d}}$ Medical Statistician, Gloucestershire Research and Development Support Unit, Gloucestershire Health \\ Authority, UK \\ e GPIAG Professor of Primary Care Respiratory Medicine, Department of Gererd Pr. Cune and Primary \\ Care, University of Aberdeen, UK \\ Received 15 June 2004; accepted jor Octope ?2014
}

\section{KEYWORLS}

Dysfunctional breathing;

Hyperventilation;

Asthma;

Epidemiology cumrnily runctional breathing problems, including symptomatic hyperventilation, may impair quality of life. Symptoms associated with functional breathing disorders have been reported as being common in secondary care settings, and can affect $29 \%$ of adults with current asthma in the community. The prevalence of dysfunctional breathing in the general adult population is unknown.

The Nijmegen Questionnaire has been reported to have useful sensitivity and specificity for diagnosing dysfunctional breathing. A cross-sectional postal survey of adults without current asthma was undertaken in a single UK general practice. The results were analysed in conjunction with a previously described survey of adults with current asthma from the same population.

The questionnaire was posted to a random sample of 300 people aged 16-65 without current asthma, and $69 \%$ were returned. $8 \%$ (95\% confidence intervals $4-12 \%$ ) had positive screening scores. Positive screening scores were more common in women $(14 \%, 7-20 \%)$ than men $(2 \%, 0-5 \%, p=0.003)$. Comparison with the previous survey showed that the prevalence of positive screening scores was higher in those with current asthma than those without (29\% vs. $8 \%, p<0.001)$.

Dysfunctional breathing may affect up to one in 10 people, and is more common in women and in people with asthma.

(c) 2004 General Practice Airways Group. Published by Elsevier Ltd. All rights reserved.

\footnotetext{
* Corresponding author. Present address: Minchinhampton Surgery, Minchinhampton, Stroud, Gloucs GL6 9JF, UK. Tel.: +44 1285 760671; fax: +44 1453731670.

E-mail address: mikethomas@doctors.org.uk (M. Thomas).
} 


\section{Introduction}

Functional breathing problems have been shown to result in significant morbidity, including respiratory symptoms such as breathlessness, chest tightness and chest pain, and non-respiratory symptoms including anxiety, light headedness and fatigue $[1,2]$. Because people frequently overbreathe or have an increased respiratory rate, this syndrome is often called the "hyperventilation syndrome". Nevertheless, people may exhibit other breathing abnormalities, such as unsteadiness and irregularity of breathing [3-5], frequent sighing [4] and a predominantly non-diaphragmatic respiratory effort [1]. Other diagnostic terms have been applied to people with symptoms produced by abnormal breathing, including 'dysfunctional breathing' [6].

The prevalence of functional breathing disorders in the community is not known accurately. We have recently reported a high prevalence of symptoms compatible with dysfunctional breathing in adults treated for asthma in the community [7] with $29 \%$ of people having positive screening scores using the Nijmegen Questionnaire [8]; over half showed a clinically relevant improvement in their disease-related health status following a short physiotherapy-based breathing retrainirg programme [9].

This study uses the Mirnegen Questionnaire (Table 1) 0 (1) ines igate the prevatercel of symptoms suggestive of dysfunchonal 5 reathing in adults without asthma, and compares this with the results from the previously published survey [7] on prevalence of symptoms in adults with asthma.

\section{Methods}

This study has a cross-sectional design. We identified 4381 adults, aged 17 to 65, from the medical records of a UK semirural general practice of 7033 patients which uses a computerised clinical and prescribing software system (EMIS, Egton Medical Information System version 2.3.0). 307 people had current asthma (defined as a clinical diagnosis of asthma and receipt of one or more prescriptions for inhaled or oral bronchodilator or prophylactic asthma medication in the previous year) and the other 4,074 did not have asthma.

A cross-sectional survey using the Nijmegen Questionnaire was performed on the adults with current asthma [7]. The Nijmegen Questionnaire is a self-complete screening instrument, and a score of $\geq 23$ has a reported sensitivity of $91 \%$ and specificity of $95 \%$ for the diagnosis of symptomatic hyperventilation [8]. A subsequent survey was performed 12 months later on a random sample of 300 of the 4,074 people without current asthma using the same questionnaire. A random sample was generated automatically using the facility incorporated in the EMnS software system. The questionairt Wos posted to these 300 people for .ien to self-complets and return, together with a covering letier explaining that a survey was being indertaken of symptoms associated with breathing problems in people with and without asthma. A Nijmegen score $\geq 23$ was used as a positive screening score. Age and sex were obtained from the patient records. Results from the two surveys were compared.

Table 1 Nijmegen Questionnaire (Please ring the score that best describes the frequency with which you experience the symptoms listed below).

\begin{tabular}{|c|c|c|c|c|c|}
\hline & Never & Seldom & Some-times & Often & Very Often \\
\hline Chest pain & 0 & 1 & 2 & 3 & 4 \\
\hline Feeling tense & 0 & 1 & 2 & 3 & 4 \\
\hline Blurred vision & 0 & 1 & 2 & 3 & 4 \\
\hline Dizziness & 0 & 1 & 2 & 3 & 4 \\
\hline Confusion or loss of touch with reality & 0 & 1 & 2 & 3 & 4 \\
\hline Fast or deep breathing & 0 & 1 & 2 & 3 & 4 \\
\hline Shortness of breath & 0 & 1 & 2 & 3 & 4 \\
\hline Tightness across chest & 0 & 1 & 2 & 3 & 4 \\
\hline Bloated sensation in stomach & 0 & 1 & 2 & 3 & 4 \\
\hline Tingling in fingers and hands & 0 & 1 & 2 & 3 & 4 \\
\hline Difficulty in breathing or taking a deep breath & 0 & 1 & 2 & 3 & 4 \\
\hline Stiffness or cramps in fingers and hands & 0 & 1 & 2 & 3 & 4 \\
\hline Tightness around the mouth & 0 & 1 & 2 & 3 & 4 \\
\hline Cold hands or feet & 0 & 1 & 2 & 3 & 4 \\
\hline Palpitations in the chest & 0 & 1 & 2 & 3 & 4 \\
\hline Anxiety & 0 & 1 & 2 & 3 & 4 \\
\hline
\end{tabular}


Table 2 Nijmegen screening questionnaire results in subjects with and without current asthma (percentages with positive screening scores, 95\% Confidence Intervals, $\mathrm{Cl}$ ).

\begin{tabular}{llllll}
\hline & $\begin{array}{l}\text { \% of mailings } \\
\text { returned }\end{array}$ & $\begin{array}{l}\text { \% }(95 \% \mathrm{Cl}) \text { of } \\
\text { subjects } \\
\text { with positive } \\
\text { screening }\end{array}$ & $\begin{array}{l}\text { Mean age }(95 \% \\
\mathrm{Cl}) \text { of subjects } \\
\text { with positive } \\
\text { screening scores }\end{array}$ & $\begin{array}{l}\text { \% }(95 \% \mathrm{Cl}) \text { of } \\
\text { male subjects } \\
\text { with positive }\end{array}$ & $\begin{array}{l}\text { sceening }(95 \% \mathrm{Cl}) \text { of } \\
\text { female subjects } \\
\text { with positive } \\
\text { screening scores }\end{array}$ \\
\hline Asthma & 71 & $29(23-35)$ & $44.8(14.7)$ & $20(11-28)$ & $35(27-43)$ \\
Non asthma & 69 & $8(4-12)$ & $44.3(13.2)$ & $2(0-5)$ & $14(7-20)$ \\
\hline
\end{tabular}

The study was approved by the local research ethics committee.

Data was entered on a computerised spreadsheet and analysed using standard SPSS software. Sex difference in the positive and negative screening groups was compared using chi-squared test. Age difference was analysed using Students $t$ test. Descriptive statistics are presented as proportions with 95\% Confidence Intervals $(\mathrm{Cl})$ and as means with Standard deviation (SD). The prevalence of positive screening scores between people with and without asthma was compared using the Mann-Whitney U-test.

\section{Results}

Of the 300 mailings, 20) questran riatires (69\%) were returned and we G uitable for analysis. 38 respondents (47\%) were male, and tite miean age was 46.6 years, standard deviation (SD) 12.2 years. The characteristics of subjects with positive screening scores in the two surveys are shown in Table 2.

17 subjects $(8 \%)$ scored $\geq 23$. More women $(15 / 110,14 \%)$ than men $(2 / 98,2 \%)$ had positive screening scores of $\geq 23$ (chi squared $=9.0, d f=1$, $p=0.003$ ) (Fig. 1). The mean age of those scoring $\geq 23$ (44.3 years, SD 13.2) was not significantly different from those scoring $<23$ (46.8 years, SD

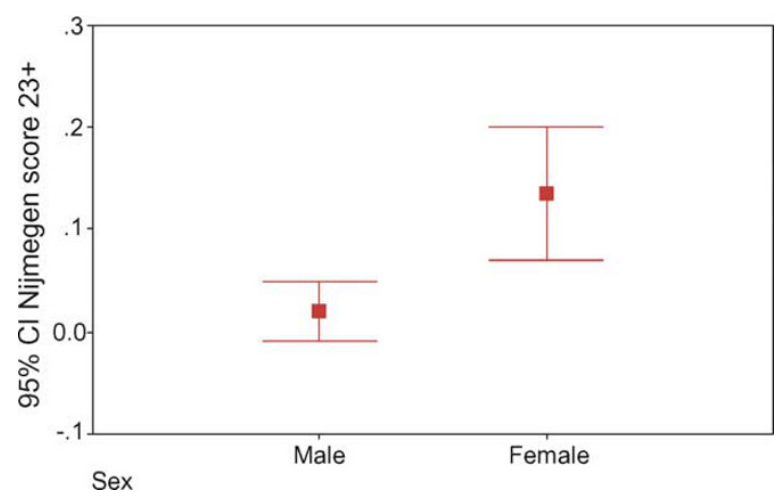

Figure 1 Proportion of positive screening scores by sex in patients without current asthma.
12.1), (difference 2.5 years, standard error of difference 3.3 years, $p=0.46$ ).

In comparison with the previous survey of people with asthma from the same population, the prevalence of positive screening scores was higher in those diagnosed and treated for asthma (29\%) than in those with no diagnosis of asthma (8\%), $p<0.001$ (Mann-Whitney U-test) (Fig. 2).

With a prevalence of positive screening scores of $29 \%$ in people with treated asthma (comprising $7 \%$ of adult population) and $8 \%$ in people without current asthma (comprising 93\% of adult population), the estimated prevalence of positive screening scores in the overall aujult population was $(8 \times 0.930+(29) \times 0.07)=9.5 \%$.

\section{Discussion}

This study demonstrates that a significant minority $(9.5 \%)$ of adults in the community have symptoms suggestive of dysfunctional breathing (positive Nijmegen Questionnaire screening scores), and that up to one in 10 adults may be experiencing potentially treatable symptoms and impairment of their quality of life.

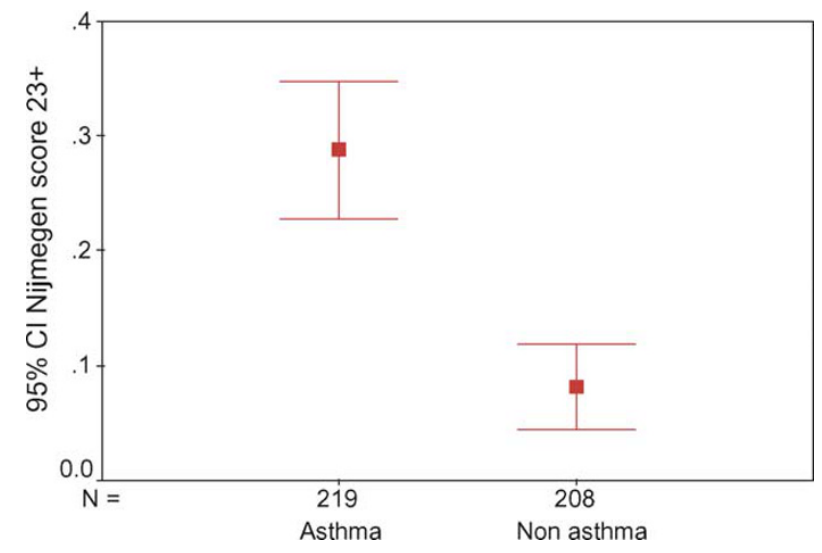

Figure 2 Proportions of positive screening scores in asthma and non-asthma patients. 
We have previously reported that positive Nijmegen Questionnaire screening scores are found in over $25 \%$ of asthma patients in the community [7]. Although the Nijmegen questionnaire was validated as a screening tool for the diagnosis of symptomatic hyperventilation in a general population and not in asthmatic populations, the demonstration that over $50 \%$ of these asthmatic patients experienced a clinically relevant improvement in their asthma-related quality of life following a brief breathing-retraining intervention [9] supports the validity of the questionnaire tool in recognising potentially treatable dysfunctional breathing in patients with asthma. In the subsequent survey reported here from a general primary care population, a group in whom the questionnaire has been validated, we found approximately one in 10 patients had positive screening scores. Positive screening scores are therefore three times as common in people diagnosed and treated for asthma, and are more common in women than men.

The Nijmegen Questionnaire [8] (Table 1) is a simple-to-use symptom-based questionnaire evaluating the frequency of 16 symptoms commonly associated with symptomatic hyperventilation and dysfunctional breathing; it is a practicable tool iii community settings as part of a general partert assessment. Since all thes symptons are nonspecific and common to biner disease procasses, it is always necessary to consider ether diagnoses and to investigate where appropriate, although the diagnosis can only be confirmed by a clinical improvement in response to successful breathing modification. The questionnaire does, however, identify people with a characteristic constellation of symptoms, including respiratory and nonrespiratory complaints, and has been reported to have sensitivity and specificity of $>90 \%$ as compared with other diagnostic methods for the 'hyperventilation syndrome' [8]. The reliability, validity and responsiveness of the Nijmegen Questionnaire (as a patient-centered diagnostic and outcome measure) as compared with ideal criteria [10] is currently incompletely described. Difficulties in validation arise from the lack of a simple 'gold-standard' diagnostic test to confirm or refute dysfunctional breathing. We have provided evidence of useful predictive validity and criterion validity (from the correlation of changes in Nijmegen Questionnaire scores with changes in disease-related quality of life) in previous work [9].

This survey occurred in a single UK general practice population, and the practice demographic profile is similar to other UK general practice populations. The response rates (of approximately
$70 \%$ in both of the surveys were reasonable and typical of such general practice postal surveys. The practice provides structured asthma care with an asthma clinic and a trained asthma nurse, but this is common in the UK. There is no reason to believe that the surveyed population or the delivery of care is atypical, although larger surveys would be needed to confirm this. This survey occurred in the same general practice population as the earlier asthma survey, and the demographic profiles of patients sent and returning questionnaires were similar in both surveys. This survey occurred 12 months after the earlier survey, and so occurred at the same time of year. Although the two groups were not surveyed simultaneously, there is no evidence of any difference between the groups other than the diagnosis of asthma, and no reason to suspect that significant demographic or morbidity change had occurred in this population over the 12-month period.

The reasons why symptoms suggestive of dysfunctional breathing should be more common in people with asthma than those without are uncertain. Possible explanations include: misdiagnosis of asthma in $P\left(C_{0}\right)\left(V_{n}\right.$ it l respiratory symptoms peinting fiom other causes; the increal:ed Uprevalence of heightened anxiety and panic disorden iolorered in people with asthma [11], abnorrnai respiratory patterns resulting from difificulties in breathing experienced by people with asthma; and potential pathogenic inflammatory effects of over-breathing [12]. Further studies are needed to confirm and elucidate this observation.

Intervention studies of breathing-retraining have indicated that non-pharmacological treatment can be used successfully to treat dysfunctional breathing in people with asthma [9] and without asthma $[13,14]$. None of the people identified in these surveys had previously received a diagnosis of dysfunctional breathing nor had they received any treatment for it. This finding suggests that there may be an unmet need in the community, with people experiencing symptoms that may potentially be helped by a simple non-pharmacological treatment.

In summary, we found that symptoms suggestive of dysfunctional breathing are not uncommon in adults in the community, particularly in women, and are three times as common in adults with asthma than in those without.

\section{Funding}

UK Royal Collage of General Practitioners Scientific Foundation Board.

\section{Conflict of interest}

None. 


\section{References}

[1] Folgering $\mathrm{H}$. The pathophysiology of hyperventilation disorder. Monalsi Arch Chest Dis 1999;54:365-71.

[2] Gardner WN. The pathophysiology of hyperventilation disorders. Chest 1996;109:516-34.

[3] Han JN, Stegen K, Simkens K, Caubergs M, Schepers R, Van den Bergh O, et al. Unsteadiness of breathing in patients with hyperventilation syndrome and anxiety disorders. Eur Respir J 1997;10:167-76.

[4] Hormbrey J, Jacobi MS, Patil CP, Saynders KB. CO2 reponse and pattern of breathing in patients with symptomatic hyperventialtion compared with asthmatic and normal subjects. Eu Respir J 1988;1:846-52.

[5] Han JN, Stegen K, Schepers R, Van den Bergh O, Woestinjne KP. Subjective symptoms and breathing pattern at rest and following hyperventilation in anxiety and somatiform disorders. J Psychosom Res 1998;45:519-32.

[6] van Dixhoorn J. Hyperventilation and dysfunctional breathing. Biological Psychology 1997;46:90-1.

[7] Thomas M, McKinley RK, Freeman E, Foy C. Prevalence of dysfunctional breathing in patients treated for asthma in primary care: cross sectional survey. BMJ 2001;322:1098-100.
[8] van Dixhoorn J, Duivenvoorden HJ. Efficacy of Nijmegen questionnaire in recognition of the hyperventilation syndrome. J Psycosom Res 1985;29:199-206.

[9] Thomas M, McKinley RK, Freeman E, Foy C, Prodger P, Price D. Breathing retraining for dysfunctional breathing in asthma- a randomised controlled trial. Thorax 2003;58:110-5.

[10] Fitzpatrick R, Davey C, Buxton MJ, Jones DR. Evaluating patient-based outcome measures for use in clinical trials. Health Technol Assessment 1998;2(14).

[11] Carr RE, Leher PM, Rausch LL, Hochron SM. Anxiety sensitivity and panic attacks in as asthmatic population. Behav Res Ther 1994;32:411-8.

[12] Davis MS, Freed AN. Repeated hyperventilation causes peripheral airways inflammation, hyperreactivity and impaired bronchodilation in dogs. Am J Respir Crit Care Med 2001;164:785-9.

[13] DeGuire S, Gevirtz R, Hawkinson D, Doxon K. Breathing retraining: a three-year follow-up study of treatment for hyperventilation syndrome and associated functional cardiac symptoms. Biofeedback Self Regul 1996;21:1918.

[14] Grossman P, De Swart JC, Defares PB. A controlled study of a breathing therapy for treatment of hyperventilation syndrome. J Psycosom Res 1985;29(1):49-58.

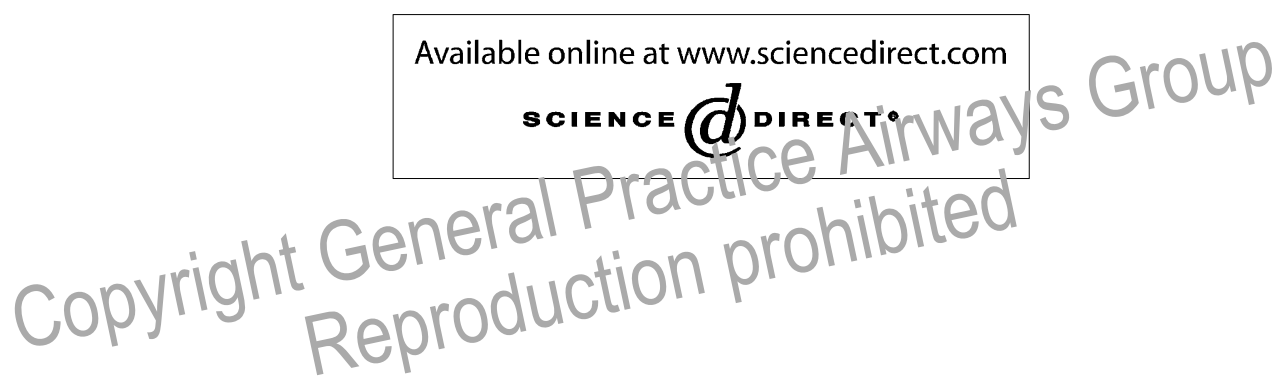

Available online at http://www.thepcrj.com 